\title{
Inverse Scattering Problem for Vector Fields and the Cauchy Problem for the Heavenly Equation
}

\author{
S. V. Manakov ${ }^{1, \S}$ and P. M. Santini ${ }^{2, \S}$ \\ ${ }^{1}$ Landau Institute for Theoretical Physics, Moscow, Russia \\ 2 Dipartimento di Fisica, Università di Roma "La Sapienza", and \\ Istituto Nazionale di Fisica Nucleare, Sezione di Roma 1 \\ Piazz.le Aldo Moro 2, I-00185 Roma, Italy

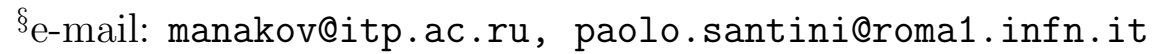

September 27, 2018

\begin{abstract}
We solve the inverse scattering problem for multidimensional vector fields and we use this result to construct the formal solution of the Cauchy problem for the second heavenly equation of Plebanski, a scalar nonlinear partial differential equation in four dimensions relevant in General Relativity, which arises from the commutation of multidimensional Hamiltonian vector fields.
\end{abstract}

\section{Introduction}

In this paper we solve the inverse scattering problem for multidimensional vector fields and we use this result to construct the formal solution of the Cauchy problem for the real second heavenly equation

$$
\theta_{t x}-\theta_{z y}+\theta_{x x} \theta_{y y}-\theta_{x y}^{2}=0, \quad \theta=\theta(x, y, z, t) \in \mathbb{R}, \quad x, y, z, t \in \mathbb{R},
$$

where subscripts denote partial derivatives.

This scalar nonlinear partial differential equation (PDE) in 4 independent variables $x, y, z, t$, introduced in [1] by Plebanski, describes the Einstein field equations that govern self-dual gravitational fields. Its 2-dimensional reduction $\theta_{z}=\theta_{t}=0$ is the Monge-Ampère equation, relevant in Differential Geometry. As we shall see in the following, the heavenly equation plays also 
a distinguished role in the theory of commuting, 2-dimensional, Hamiltonian dynamical systems.

The heavenly equation, together with the equations for the self-dual YangMills (SDYM) fields [2], are perhaps the most distinguished examples of nonlinear PDEs in more than three independent variables arising as commutativity conditions of linear operators [3], and therefore amenable, in principle, to exact treatments based on the spectral theory of those operators [4], $[5]$. If the SDYM equations are considered on an abstract Lie algebra, then the heavenly equation can actually be interpreted as a distinguished realization of the SDYM equations, corresponding to the Lie algebra of divergence free vector fields independent of the SDYM coordinates [6]. Equation (11) has been investigated within the twistor approach in [7, [8]. A bi-Hamiltonian formulation and a hodograph transformation for (11) have been recently constructed in [9] and [10]; a nonlinear $\bar{\partial}$-dressing and a generating equation for its hierarchy can be found in [11.

The present paper is a simplified version of the manuscript 12 .

\section{Integrable PDEs in arbitrary dimensions and the heavenly equation}

It is known that the commutation of multidimensional vector fields leads to nonlinear first order multidimensional PDEs (see, f.i., [3]). In this spirit, we derive now a basic class of integrable nonlinear PDEs in arbitrary dimensions possessing, as distinguished reduction, the heavenly equation (11).

Consider the following pair of vector fields

$$
\hat{L}_{i}=\partial_{t_{i}}+\lambda \partial_{z_{i}}+\sum_{k=1}^{N} u_{i}^{k} \partial_{x_{k}}=\partial_{t_{i}}+\lambda \partial_{z_{i}}+\vec{u}_{i} \cdot \nabla_{\vec{x}}, \quad i=1,2
$$

where $\partial_{x}$ denotes partial differentiation with respect to the generic variable $x, \vec{x}=\left(x_{1}, . ., x_{N}\right), \nabla_{\vec{x}}=\left(\partial_{x_{1}}, . ., \partial_{x_{N}}\right), \vec{u}_{i}=\left(u_{i}^{1}, . ., u_{i}^{N}\right), i=1,2, \lambda$ is a complex parameter and the vector coefficients $\vec{u}_{i}$ depend on the independent variables $t_{i}, z_{i}, x_{k}, i=1,2, k=1, . ., N$, but not on $\lambda$. The existence of a common eigenfunction $f$ for the operators $\hat{L}_{1}$ and $\hat{L}_{2}$ :

$$
\hat{L}_{1} f=\hat{L}_{2} f=0
$$


implies their commutation, $\forall \lambda$ :

$$
\left[\hat{L}_{1}, \hat{L}_{2}\right]=0
$$

which is equivalent to the following determined system of $2 N$ first order quasi-linear PDEs in $(4+N)$ dimensions for the $2 N$ fields $\vec{u}_{1}, \vec{u}_{2}$ :

$$
\begin{aligned}
& {\overrightarrow{u_{1}}}_{z_{2}}={\overrightarrow{u_{2} z_{1}}}, \\
& \overrightarrow{u_{1 t_{2}}}-\overrightarrow{u_{2 t_{1}}}+\left(\overrightarrow{u_{2}} \cdot \nabla_{\vec{x}}\right) \vec{u}_{1}-\left(\vec{u}_{1} \cdot \nabla_{\vec{x}}\right) \vec{u}_{2}=\overrightarrow{0} .
\end{aligned}
$$

Parametrizing the first set of equations in terms of the vector potential $\vec{U}$

$$
\vec{u}_{i}=\vec{U}_{z_{i}}, \quad i=1,2
$$

one obtains the following determined system of $N$ nonlinear PDEs for the $N$ dependent variables $\vec{U}$ in $(4+N)$ dimensions:

$$
\vec{U}_{t_{1} z_{2}}-\vec{U}_{t_{2} z_{1}}+\left(\vec{U}_{z_{1}} \cdot \nabla_{\vec{x}}\right) \vec{U}_{z_{2}}-\left(\vec{U}_{z_{2}} \cdot \nabla_{\vec{x}}\right) \vec{U}_{z_{1}}=\overrightarrow{0}
$$

This system admits a natural reduction; indeed, applying the operator $\nabla_{\vec{x}} \cdot$ to equations (17), one obtains

$$
\left[\partial_{t_{1}} \partial_{z_{2}}-\partial_{t_{2}} \partial_{z_{1}}+\left(\vec{U}_{z_{1}} \cdot \nabla_{\vec{x}}\right) \partial_{z_{2}}-\left(\vec{U}_{z_{2}} \cdot \nabla_{\vec{x}}\right) \partial_{z_{1}}\right]\left(\nabla_{\vec{x}} \cdot \vec{U}\right)=0,
$$

from which one infers that the condition

$$
\nabla_{\vec{x}} \cdot \vec{U}=0
$$

is an admissible reduction for equation (7), implying that the condition of zero-divergence:

$$
\nabla_{\vec{x}} \cdot \vec{u}_{i}=0, \quad i=1,2
$$

is an admissible constraint for the vectors $\vec{u}_{i}, i=1,2$.

From now on, we concentrate our attention on the following important example:

$$
N=2, \quad z_{i}=x_{i}, \quad i=1,2
$$

and we make the following change of notation for the remaining 4 variables:

$$
t_{1}=z, t_{2}=t, \quad x_{1}=x, \quad x_{2}=y \text {. }
$$


Then the system (17) reduces to the following determined system of two PDEs in 4 dimensions:

$$
\begin{aligned}
& \vec{U}_{t x}-\vec{U}_{z y}+\left(\vec{U}_{y} \cdot \nabla_{\vec{x}}\right) \vec{U}_{x}-\left(\vec{U}_{x} \cdot \nabla_{\vec{x}}\right) \vec{U}_{y}=\overrightarrow{0}, \\
& \vec{U} \in \mathbb{R}^{2}, \quad \vec{x}=(x, y), \quad \nabla_{\vec{x}}=\left(\partial_{x}, \partial_{y}\right),
\end{aligned}
$$

corresponding to the Lax pair:

$$
\begin{array}{ll}
\hat{L}_{1}=\partial_{z}+\lambda \partial_{x}+\vec{u}_{1} \cdot \nabla_{\vec{x}}, & \vec{u}_{1}=\vec{U}_{x}, \\
\hat{L}_{2}=\partial_{t}+\lambda \partial_{y}+\vec{u}_{2} \cdot \nabla_{\vec{x}}, & \vec{u}_{2}=\vec{U}_{y} .
\end{array}
$$

In this case, the zero-divergence reduction (10) makes the two vector fields $\vec{u}_{i} \cdot \nabla_{\vec{x}}$ Hamiltonian, allowing for the introduction of two Hamiltonians $H_{i}, i=$ 1,2 such that:

$$
\vec{u}_{i}=\left(H_{i y},-H_{i x}\right), \quad i=1,2
$$

which, due to $(5 \mathrm{~b})$, are parametrized by a single potential $\theta$ :

$$
\begin{aligned}
& H_{i}=\theta_{x_{i}}, \quad \vec{U}=\left(\theta_{y},-\theta_{x}\right), \\
& \vec{u}_{1}=\left(\theta_{x y},-\theta_{x x}\right), \quad \vec{u}_{2}=\left(\theta_{y y},-\theta_{x y}\right) .
\end{aligned}
$$

Then the compatible linear problems (31), (14) can be written down as Hamilton equations with respect to the times $z, t$ :

$$
\begin{aligned}
& f_{z}=\left\{H_{1}+\lambda y, f\right\}_{\vec{x}}, \\
& f_{t}=\left\{H_{2}-\lambda x, f\right\}_{\vec{x}},
\end{aligned}
$$

where $\{\cdot, \cdot\}_{\vec{x}}$ is the Poisson bracket with respect to the variables $x, y$ :

$$
\{f, g\}_{\vec{x}}=f_{x} g_{y}-f_{y} g_{x}
$$

and the nonlinear system (13) reduces to the heavenly equation in Hamiltonian form

$$
\theta_{t x}-\theta_{z y}+\left\{\theta_{x}, \theta_{y}\right\}_{\vec{x}}=\text { constant }
$$

equivalent to (11) after choosing the constant to be zero.

\section{IST for the nonlinear PDEs (13) and (1)}

Since the Lax pair (14) is made of vector fields, Hamiltonian in the heavenly reduction (10), (11), the eigenfunctions satisfy the following basic properties, 
which will introduce important novelties in the Inverse Scattering Transform (IST).

1) The space of eigenfunctions is a ring: if $f_{1}, f_{2}$ are two solutions of the Lax pair (14), then an arbitrary differentiable function $F\left(f_{1}, f_{2}\right)$ of them is a solution of (14).

2) In the heavenly (Hamiltonian) reduction (10), (11), the space of eigenfunctions is also a Lie algebra, whose Lie bracket is the natural Poisson bracket (18): if $f_{1}, f_{2}$ are two solutions of the Lax pair (14), then their Poisson bracket $\left\{f_{1}, f_{2}\right\}_{(x, y)}$ is also a solution of (14).

Now we consider the Cauchy problem for the system (13) and for its heavenly reduction (1), within the class of rapidly decreasing real potentials $u_{i}^{j}$ :

$$
\begin{aligned}
& u_{i}^{j} \rightarrow 0, \quad\left(x^{2}+y^{2}+z^{2}\right) \rightarrow \infty, \\
& u_{i}^{j} \in \mathbb{R}, \quad(x, y, z) \in \mathbb{R}^{3}, \quad t>0,
\end{aligned}
$$

interpreting $t$ as time and the other three variables $x, y, z$ as space variables.

To solve such a Cauchy problem by the IST method (see, f.i., 4, [5]), we construct the IST for the operator $\hat{L}_{1}$ in (14), within the class of rapidly decreasing real potentials, interpreting the operator $\hat{L}_{2}$ in (14) as the time operator. The formalism presented here can be generalized to the vector fields (2) in a straightforward way.

\subsection{Basic eigenfunctions}

The localization (20) of the vector potential $\vec{u}_{1}$ implies that, if $f$ is a solution of $\hat{L}_{1} f=0$, then

$$
\begin{aligned}
& f(\vec{x}, z, \lambda) \rightarrow f_{ \pm}(\vec{\xi}, \lambda), \quad z \rightarrow \pm \infty \\
& \vec{\xi}:=\vec{x}-(\lambda, 0) z ;
\end{aligned}
$$

i.e., asymptotically, $f$ is an arbitrary function of $(x-\lambda z), y$ and $\lambda$.

A central role in the theory is played by the real Jost eigenfunctions $\varphi_{1,2}(\vec{x}, z, \lambda)$, the solutions of $\hat{L}_{1} \varphi_{1,2}=0$ uniquely defined by the asymptotics

$$
\varphi_{1}(\vec{x}, z, \lambda) \rightarrow x-\lambda z \equiv \xi, \quad \varphi_{2}(\vec{x}, z, \lambda) \rightarrow y, \quad z \rightarrow-\infty .
$$

In this paper we often use the compact vector notation: $\vec{f}=\left(f_{1}, f_{2}\right)^{T}$. Then:

$$
\vec{\varphi}(\vec{x}, z, \lambda) \equiv\left(\begin{array}{c}
\varphi_{1}(\vec{x}, z, \lambda) \\
\varphi_{2}(\vec{x}, z, \lambda)
\end{array}\right) \rightarrow\left(\begin{array}{c}
\xi \\
y
\end{array}\right) \equiv \vec{\xi}, \quad z \rightarrow-\infty
$$


The Jost eigenvector $\vec{\varphi}$ is equivalently characterized by the integral equation

$$
\begin{aligned}
& \vec{\varphi}(\vec{x}, z, \lambda)+ \\
& \int_{\mathbb{R}^{3}} d \vec{x}^{\prime} d z^{\prime} G\left(\vec{x}-\vec{x}^{\prime}, z-z^{\prime} ; \lambda\right)\left(\vec{u}_{1}\left(\vec{x}^{\prime}, z^{\prime}\right) \cdot \nabla_{\vec{x}^{\prime}}\right) \vec{\varphi}\left(\vec{x}^{\prime}, z^{\prime}, \lambda\right)=\vec{\xi},
\end{aligned}
$$

in terms of the Jost Green's function

$$
G(\vec{x}, z ; \lambda)=\theta(z) \delta(x-\lambda z) \delta(y) .
$$

A crucial role in the IST for the vector field $\hat{L}_{1}$ is also played by the analytic eigenfunctions $\vec{\psi}_{ \pm}(\vec{x}, z, \lambda)$, the solutions of $\hat{L}_{1} \vec{\psi}_{ \pm}=\overrightarrow{0}$ satisfying the integral equations

$$
\vec{\psi}_{ \pm}(\vec{x}, z, \lambda)+\int_{\mathbb{R}^{3}} d \vec{x}^{\prime} d z^{\prime} G_{ \pm}\left(\vec{x}-\vec{x}^{\prime}, z-z^{\prime} ; \lambda\right)\left(\vec{u}_{1}\left(\vec{x}^{\prime}, z^{\prime}\right) \cdot \nabla_{\vec{x}^{\prime}}\right) \vec{\psi}_{ \pm}\left(\vec{x}^{\prime}, z^{\prime}, \lambda\right)=\vec{\xi},
$$

where $G_{ \pm}$are the analytic Green's functions

$$
G_{ \pm}(\vec{x}, z ; \lambda)= \pm \frac{\delta(y)}{2 \pi i[x-(\lambda \pm i \epsilon) z]} .
$$

The analyticity properties of $G_{ \pm}(\vec{x}, z, \lambda)$ in the complex $\lambda$ - plane imply that $\vec{\psi}_{+}(\vec{x}, z, \lambda)$ and $\vec{\psi}_{-}(\vec{x}, z, \lambda)$ are analytic, respectively, in the upper and lower halves of the complex $\lambda$ - plane, with the following asymptotics, for large $\lambda$ :

$$
\vec{\psi}_{ \pm}(\vec{x}, z, \lambda)=\vec{\xi}+\frac{\vec{Q}_{ \pm}(\vec{x}, z)}{\lambda}+O\left(\lambda^{-2}\right) . \quad|\lambda|>>1,
$$

where:

$$
\vec{Q}_{ \pm}(\vec{x}, z)= \pm P \int_{\mathbb{R}^{2}} \frac{d x^{\prime} d z^{\prime}}{2 \pi i\left(z-z^{\prime}\right)} \vec{u}_{1}\left(x^{\prime}, y, z^{\prime}\right)-\frac{1}{2}\left(\int_{-\infty}^{x}-\int_{x}^{\infty}\right) d x^{\prime} \vec{u}_{1}\left(x^{\prime}, y, z\right),
$$

entailing that

$$
\vec{u}_{1}(\vec{x}, z)=-\vec{Q}_{ \pm x}(\vec{x}, z) .
$$

It is important to remark that the analytic Green's functions (27) exhibit the following asymptotics for $z \rightarrow \pm \infty$ :

$$
\begin{array}{ll}
G_{ \pm}(\vec{x}, z ; \lambda) \rightarrow \pm \frac{\delta(y)}{2 \pi i[\xi \mp i \epsilon]}, & z \rightarrow+\infty, \\
G_{ \pm}(\vec{x}, z ; \lambda) \rightarrow \pm \frac{\delta(y)}{2 \pi i[\xi \pm i \epsilon]}, & z \rightarrow-\infty,
\end{array}
$$

It follows that the $z=+\infty$ asymptotics of $\vec{\psi}_{+}$and $\vec{\psi}_{-}$are analytic respectively in the lower and upper halves of the complex plane $\xi$, while the $z=-\infty$ asymptotics of $\vec{\psi}_{+}$and $\vec{\psi}_{-}$are analytic respectively in the upper and lower halves of the complex plane $\xi$. This mechanism was first observed in [13]. 


\subsection{Spectral data}

The $z=+\infty$ limit of $\vec{\varphi}$ defines the scattering vector $\vec{\sigma}$ of $\hat{L}_{1}$ :

$$
\lim _{z \rightarrow+\infty} \vec{\varphi}(\vec{x}, z ; \lambda) \equiv \overrightarrow{\mathcal{S}}(\vec{\xi}, \lambda)=\vec{\xi}+\vec{\sigma}(\vec{\xi}, \lambda) .
$$

The direct problem is the mapping from the real vector potential $\vec{u}_{1}$, function of the three real variables $(\vec{x}, z)$, to the real scattering vector $\vec{\sigma}$, function of the three real variables $(\vec{\xi}, \lambda)$. Then the counting is consistent. The impact of the Heavenly constraint (10), (11) on the spectral data will be discussed in Section 3.5.

The Jost solutions $\varphi_{1,2}$ form, together with the constant eigenfunction $\lambda$, a basis in the space of the eigenfunctions of $\hat{L}_{1}$ (which is a ring). The representation of the analytic eigenfunctions $\vec{\psi}_{ \pm}$in terms of $\vec{\varphi}$ yields:

$$
\vec{\psi}_{ \pm}(\vec{x}, z, \lambda)=\overrightarrow{\mathcal{K}}_{ \pm}(\vec{\varphi}(\vec{x}, z, \lambda), \lambda)=\vec{\varphi}(\vec{x}, z, \lambda)+\vec{\chi}_{ \pm}(\vec{\varphi}(\vec{x}, z, \lambda), \lambda),
$$

and this formula defines the spectral data $\vec{\chi}_{ \pm}$. Since the $z \rightarrow-\infty$ limit of (33) reads:

$$
\lim _{z \rightarrow-\infty} \vec{\psi}_{ \pm}-\vec{\xi}=\vec{\chi}_{ \pm}(\vec{\xi}, \lambda)
$$

the above analyticity properties of the LHS of (34) in the complex $\xi$ - plane imply that $\vec{\chi}_{+}(\vec{\xi}, \lambda)$ and $\vec{\chi}_{-}(\vec{\xi}, \lambda)$ are analytic respectively in the upper and lower halves of the complex plane $\xi$, decaying at $\xi \sim \infty$ like $O\left(\xi^{-1}\right)$. Therefore their Fourier transforms $\tilde{\vec{\chi}}_{+}(\vec{\omega}, \lambda)$ and $\tilde{\vec{\chi}}_{-}(\vec{\omega}, \lambda)$ have support respectively on the positive and negative real $\omega_{1}$ semi-axes.

The spectral data $\vec{\chi}_{ \pm}$can be constructed from the scattering vector $\vec{\sigma}$ through the solution of the following linear integral equations

$$
\begin{aligned}
& \tilde{\vec{\chi}}_{+}(\vec{\omega}, \lambda)+\theta\left(\omega_{1}\right)\left(\tilde{\vec{\sigma}}(\vec{\omega}, \lambda)+\int_{\mathbb{R}^{2}} d \vec{\eta} \tilde{\vec{\chi}}_{+}(\vec{\eta}, \lambda) Q(\vec{\eta}, \vec{\omega}, \lambda)\right)=\overrightarrow{0}, \\
& \tilde{\vec{\chi}}_{-}(\vec{\omega}, \lambda)+\theta\left(-\omega_{1}\right)\left(\tilde{\vec{\sigma}}(\vec{\omega}, \lambda)+\int_{\mathbb{R}^{2}} d \vec{\eta} \tilde{\vec{\chi}}_{-}(\vec{\eta}, \lambda) Q(\vec{\eta}, \vec{\omega}, \lambda)\right)=\overrightarrow{0},
\end{aligned}
$$

involving the Fourier transforms $\tilde{\vec{\sigma}}$ and $\tilde{\vec{\chi}}_{ \pm}$of $\vec{\sigma}$ and $\vec{\chi}_{ \pm}$:

$$
\tilde{\vec{\sigma}}(\vec{\omega}, \lambda)=\int_{\mathbb{R}^{2}} d \vec{\xi} \vec{\sigma}(\vec{\xi}, \lambda) e^{-i \vec{\omega} \cdot \vec{\xi}}, \quad \tilde{\vec{\chi}}_{ \pm}(\vec{\omega}, \lambda)=\int_{\mathbb{R}^{2}} d \vec{\xi} \vec{\chi}_{ \pm}(\vec{\xi}, \lambda) e^{-i \vec{\omega} \cdot \vec{\xi}}
$$

and the kernel:

$$
Q(\vec{\eta}, \vec{\omega}, \lambda)=\int_{\mathbb{R}^{2}} \frac{d \vec{\xi}}{(2 \pi)^{2}} e^{i(\vec{\eta}-\vec{\omega}) \cdot \vec{\xi}}\left[e^{i \vec{\eta} \cdot \vec{\sigma}(\vec{\xi}, \lambda)}-1\right]
$$


To prove this result, one first evaluates (B3) at $z=+\infty$, obtaining

$$
\lim _{z \rightarrow \infty} \vec{\psi}_{ \pm}-\vec{\xi}=\vec{\sigma}(\vec{\xi}, \lambda)+\vec{\chi}_{ \pm}(\vec{\xi}+\vec{\sigma}(\vec{\xi}, \lambda), \lambda) .
$$

Applying the integral operator $\int_{\mathbb{R}^{2}} d \vec{\xi} e^{-i \vec{\omega} \cdot \vec{\xi}}$. for $\omega_{1}>0$ and $\omega_{1}<0$ respectively to equations (38) + and $\left(\frac{\mathbb{R}^{2}}{38}\right)_{-}$, using the above analyticity properties and the Fourier representations of $\vec{\chi}_{ \pm}$and $\vec{\sigma}$, one obtains equations (35).

We end this section remarking that the reality of the potentials: $\vec{u}_{1} \in \mathbb{R}^{2}$ implies that, for $\lambda \in \mathbb{R}, \bar{\varphi}=\vec{\varphi}, \overrightarrow{\vec{\psi}}_{+}=\vec{\psi}_{-}$; consequently: $\overline{\vec{\sigma}}=\vec{\sigma}, \overline{\vec{\chi}}_{+}=\vec{\chi}_{-}$.

\subsection{Inverse Problem}

An inverse problem can be constructed from equations (B3). Subtracting $\vec{\xi}$ from equations (33) - and $(\underline{33})_{+}$, applying respectively the analyticity projectors $\hat{P}_{+}$and $\hat{P}_{-}$:

$$
\hat{P}_{ \pm} \equiv \pm \frac{1}{2 \pi i} \int_{\mathbb{R}} \frac{d \lambda^{\prime}}{\lambda^{\prime}-(\lambda \pm i \epsilon)}
$$

and adding up the resulting equations, one obtains the following nonlinear integral equation for the Jost eigenfunction $\vec{\varphi}$ :

$$
\begin{aligned}
& \vec{\varphi}(\vec{x}, z, \lambda)+\frac{1}{2 \pi i} \int_{\mathbb{R}} \frac{d \lambda^{\prime}}{\lambda^{\prime}-(\lambda+i \epsilon)} \vec{\chi}_{-}\left(\vec{\varphi}\left(\vec{x}, z, \lambda^{\prime}\right), \lambda^{\prime}\right)- \\
& \frac{1}{2 \pi i} \int_{\mathbb{R}} \frac{d \lambda^{\prime}}{\lambda^{\prime}-(\lambda-i \epsilon)} \vec{\chi}_{+}\left(\vec{\varphi}\left(\vec{x}, z, \lambda^{\prime}\right), \lambda^{\prime}\right)=\vec{\xi} .
\end{aligned}
$$

Given the spectral data $\vec{\chi}_{ \pm}$, one reconstructs the eigenfunction $\vec{\varphi}$ from (40), the analytic eigenfunctions from (33), and $\vec{u}_{1}$ from equation (301). This inversion procedure was first introduced in [14.

\section{$3.4 t$-evolution of the spectral data}

As the potentials $\vec{u}_{1,2}$ evolve in time according to equation (13), the $t$ dependence of the spectral data $\vec{\sigma}$ and $\vec{\chi}_{ \pm}$, defined in (32) and (133), is described by the equation:

$$
\begin{aligned}
& \vec{\sigma}(\vec{\xi}, \lambda, t)=\vec{\sigma}(\vec{\xi}-(0, \lambda) t, \lambda, 0), \\
& \vec{\chi}_{ \pm}(\vec{\xi}, \lambda, t)=\vec{\chi}_{ \pm}(\vec{\xi}-(0, \lambda) t, \lambda, 0) .
\end{aligned}
$$

To prove it, we first observe that

$$
\phi_{1}(\vec{x}, z, \lambda, t) \equiv \varphi_{1}(\vec{x}, z, \lambda, t), \quad \phi_{2}(\vec{x}, z, \lambda, t) \equiv \varphi_{2}(\vec{x}, z, \lambda, t)-\lambda t
$$


are, together with $\lambda$, a basis of common Jost eigenfunctions of $\hat{L}_{1}$ and $\hat{L}_{2}$. The $y=+\infty$ limit of equation $\hat{L}_{2} \vec{\phi}=\overrightarrow{0}$ yields $\vec{\sigma}_{t}+\lambda \vec{\sigma}_{y}=\overrightarrow{0}$, whose solution is (41). Analogously,

$$
\pi_{ \pm_{1}}(\vec{x}, z, \lambda, t) \equiv \psi_{ \pm_{1}}(\vec{x}, z, \lambda, t), \quad \pi_{ \pm_{2}}(\vec{x}, z, \lambda, t) \equiv \psi_{ \pm_{2}}(\vec{x}, z, \lambda, t)-\lambda t
$$

are a basis of common analytic eigenfunctions of $\hat{L}_{1}$ and $\hat{L}_{2}$; therefore

$$
\pi_{ \pm 1}=\check{\mathcal{K}}_{ \pm 1}(\vec{\phi}, \lambda), \quad \pi_{ \pm 2}=\check{\mathcal{K}}_{ \pm 2}(\vec{\phi}, \lambda)
$$

for some functions $\check{\mathcal{K}}_{ \pm 1,2}$ depending on $(\vec{x}, z, t)$ only through $\vec{\phi}$. Comparing, at $t=0$, these equations with equations (33), one expresses $\check{\mathcal{K}}_{ \pm 1,2}$ in terms of $\mathcal{K}_{ \pm 1,2}$, obtaining equations (41]).

\subsection{The heavenly reduction}

In the heavenly (Hamiltonian) reduction (10), (11), the transformations $\vec{\xi} \rightarrow$ $\overrightarrow{\mathcal{S}}(\vec{\xi}, \lambda), \vec{\xi} \rightarrow \overrightarrow{\mathcal{K}}_{ \pm}(\vec{\xi}, \lambda)$ are constrained to be canonical:

$$
\left\{\mathcal{S}_{1}, \mathcal{S}_{2}\right\}_{\vec{\xi}}=\left\{\mathcal{K}_{ \pm 1}, \mathcal{K}_{ \pm 2}\right\}_{\vec{\xi}}=1
$$

or, in terms of $\vec{\sigma}(\xi, y, \lambda)$ and $\vec{\chi}_{ \pm}(\xi, y, \lambda)$ :

$$
\sigma_{1 \xi}+\sigma_{2 y}+\left\{\sigma_{1}, \sigma_{2}\right\}_{\vec{\xi}}=\chi_{ \pm 1 \xi}+\chi_{ \pm 2 y}+\left\{\chi_{ \pm 1}, \chi_{ \pm 2}\right\}_{\vec{\xi}}=0 .
$$

To prove it, we recall that the Poisson bracket of the eigenfunctions $\varphi_{1}$ and $\varphi_{2}$ is also an eigenfunction: $\hat{L}_{1}\left\{\varphi_{1}, \varphi_{2}\right\}_{\vec{x}}=0$. Using the asymptotics (22), one infers that $\left\{\varphi_{1}, \varphi_{2}\right\}_{\vec{x}} \rightarrow 1$, at $z \rightarrow-\infty$; therefore, by uniqueness, $\left\{\varphi_{1}, \varphi_{2}\right\}_{\vec{x}}=1$. Evaluating now this Poisson bracket at $z=+\infty$ and using (32), one obtains the constraint (45) for $\overrightarrow{\mathcal{S}}$. We also observe that the eigenfunctions $\left\{\psi_{+1}, \psi_{+2}\right\}_{\vec{x}}$ and $\left\{\psi_{-1}, \psi_{-2}\right\}_{\vec{x}}$ are analytic in the upper and lower halves of the $\lambda$-plane and go to 1 at $|\lambda| \rightarrow \infty$. Since 1 is also an eigenfunction, by uniqueness they are identically 1: $\left\{\psi_{ \pm_{1}}, \psi_{ \pm_{2}}\right\}_{\vec{x}}=1$. Therefore, from the equations:

$$
\left\{\psi_{ \pm 1}, \psi_{ \pm_{2}}\right\}_{\vec{x}}=\left\{\mathcal{K}_{ \pm 1}, \mathcal{K}_{ \pm_{2}}\right\}_{\left(\varphi_{1}, \varphi_{2}\right)}\left\{\varphi_{1}, \varphi_{2}\right\}_{\vec{x}}=1
$$

consequence of (33), one infers the constraints (45) for $\overrightarrow{\mathcal{K}}_{ \pm}$. 


\section{Commuting $\lambda$-families of dynamical systems}

It is well-known (see, f.i., [15]) that linear first order PDEs like (3) are intimately related to systems of ordinary differential equations describing their characteristic curves. The vector fields $\hat{L}_{1,2}$ (14) are associated with the following two $\lambda$-families of commuting dynamical systems:

$$
\begin{aligned}
& \hat{L}_{1}: \quad \frac{d \vec{x}}{d z}=(\lambda, 0)+\vec{u}_{1}(\vec{x}, z, t), \\
& \hat{L}_{2}: \quad \frac{d \vec{x}}{d t}=(0, \lambda)+\vec{u}_{2}(\vec{x}, z, t) .
\end{aligned}
$$

In the heavenly reduction (10), (11), they read:

$$
\begin{aligned}
& \hat{L}_{1}: \quad \frac{d \vec{x}}{d z}+\left\{H_{1}+\lambda y, \vec{x}\right\}_{\vec{x}}=\overrightarrow{0}, \\
& \hat{L}_{2}: \quad \frac{d \vec{x}}{d t}+\left\{H_{2}-\lambda x, \vec{x}\right\}_{\vec{x}}=\overrightarrow{0} .
\end{aligned}
$$

Therefore: the heavenly equation characterizes the commutation, $\forall \lambda$, of two $\lambda$-families of Hamiltonian dynamical systems, with Hamiltonians:

$$
\tilde{H}_{1}=H_{1}(\vec{x}, z, t)+\lambda y, \quad \tilde{H}_{2}=H_{2}(\vec{x}, z, t)-\lambda x .
$$

There is a deep connection between the above IST and the $z$-scattering theory for the commuting flows (48) and (49). Let $\vec{\phi}(\vec{x}, z, \lambda, t)$ be the common eigenfunctions of $\hat{L}_{1}$ and $\hat{L}_{2}$ defined in (42); then, solving the system $\vec{\omega}=$ $\vec{\phi}(\vec{x}, z, \lambda, t)$ with respect to $x$ and $y$ (assuming local invertibility), one obtains the following common solution of the commuting flows (48):

$$
\vec{\omega}=\vec{\phi}(\vec{x}, z, \lambda, t) \Leftrightarrow \vec{x}=\vec{r}(z, t, \lambda, \vec{\omega}) \sim\left(\begin{array}{c}
\lambda z \\
\lambda t
\end{array}\right)+\vec{\omega}, \quad z \sim-\infty .
$$

The $z=+\infty$ limit of the solution $\vec{r}(z, t, \lambda, \vec{\omega})$ :

$$
\vec{x}=\vec{r}(z, t, \lambda, \vec{\omega}) \sim\left(\begin{array}{c}
\lambda z \\
\lambda t
\end{array}\right)+\vec{\Omega}(\vec{\omega}, \lambda), \quad y \sim+\infty
$$

defines the scattering vector $\vec{\Delta}(\vec{\omega})=\vec{\Omega}(\vec{\omega})-\vec{\omega}$ of (48), which is connected to the IST data $\overrightarrow{\mathcal{S}}$ by inverting the system $\vec{\omega}=\overrightarrow{\mathcal{S}}(x-\lambda z, y-\lambda t, \lambda)$ with respect to $x$ and $y$ :

$$
\vec{\omega}=\overrightarrow{\mathcal{S}}(x-\lambda z, y-\lambda t, \lambda, 0) \quad \Leftrightarrow \quad \vec{x}=\left(\begin{array}{c}
\lambda z \\
\lambda t
\end{array}\right)+\vec{\Omega}(\vec{\omega}, \lambda) .
$$


In the heavenly reduction, the transformation $\vec{\omega} \rightarrow \vec{\Omega}(\vec{\omega}, \lambda)$ is clearly canonical: $\left\{\Omega_{1}, \Omega_{2}\right\}_{\vec{\omega}}=1$, and the scattering vector $\vec{\Delta}$ exhibits the following constraint:

$$
\Delta_{1 \omega_{1}}+\Delta_{2 \omega_{2}}+\left\{\Delta_{1}, \Delta_{2}\right\}_{\vec{\omega}}=0 .
$$

The above IST theory allows one to reconstruct, from the scattering vector $\vec{\Delta}$, the potentials $\vec{u}_{1,2}$ of the dynamical systems (48) and (49).

\section{Other inverse problems}

Due to the ring property of the space of eigenfunctions, there are other distinguished ways to do the inverse problem.

\subsection{A nonlinear RH problem}

We begin with a more traditional (nonlinear) Riemann-Hilbert (RH) prob-

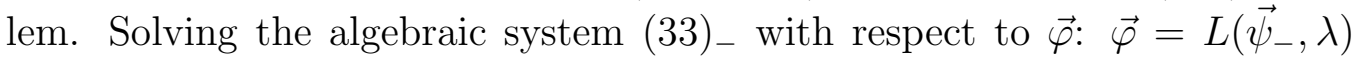
(assuming local invertibility) and replacing this expression in the algebraic system $(33)_{+}$, one obtains the representation of the analytic eigenfunction $\vec{\psi}_{+}$in terms of the analytic eigenfunction $\vec{\psi}_{-}$:

$$
\vec{\psi}_{+}=\overrightarrow{\mathcal{R}}\left(\vec{\psi}_{-}, \lambda\right)=\vec{\psi}_{-}+\vec{R}\left(\vec{\psi}_{-}, \lambda\right), \quad \lambda \in \mathbb{R}
$$

which defines a vector nonlinear $R H$ problem on the real $\lambda$ axis. The $\mathrm{RH}$ data $\vec{R}$ are therefore constructed from the data $\vec{\chi}_{ \pm}$by algebraic manipulation. Viceversa, given the RH data $\vec{R}$, one constructs the solutions $\vec{\psi}_{ \pm}$of the nonlinear RH problem (55) and, via the asymptotics (28), the potential $\vec{u}_{1}$.

As for the other spectral data, one can show that the $t$-dependence of $\vec{R}$ is described by $\vec{R}(\vec{\xi}, \lambda, t)=\vec{R}(\vec{\xi}-(0, \lambda) t, \lambda, 0)$, and the reality constraint takes the following form, for $\lambda \in \mathbb{R}: \overrightarrow{\mathcal{R}}(\overrightarrow{\mathcal{R}}(\overline{\vec{\zeta}}, \lambda), \lambda)=\vec{\zeta}, \forall \vec{\zeta}$. At last, the heavenly constraint reads $\left\{\mathcal{R}_{1}, \mathcal{R}_{2}\right\}_{\vec{\zeta}}=1$, or, in terms of $\vec{R}(\vec{\zeta}, \lambda)$ :

$$
R_{1 \zeta_{1}}+R_{2 \zeta_{2}}+\left\{R_{1}, R_{2}\right\}_{\vec{\zeta}}=0
$$

\subsection{Linearization of the inverse problem}

It is possible to construct a linear version of the inverse problem of Section 3.3 by exponentiating the Jost and analytic eigenfunctions used so far. Consider 
the following scalar functions:

$$
\Phi(\vec{x}, z, \lambda, \vec{\alpha}) \equiv e^{i \vec{\alpha} \cdot \vec{\varphi}(\vec{x}, z, \lambda)}, \quad \Psi_{ \pm}(\vec{x}, z, \lambda, \vec{\alpha}) \equiv e^{i \vec{\alpha} \cdot \vec{\psi}_{ \pm}(\vec{x}, z, \lambda)}, \quad \vec{\alpha} \in \mathbb{R}^{2}
$$

Due to the ring property of the space of eigenfunctions, also $\Phi$ and $\Psi_{ \pm}$are eigenfunctions; $\Phi$ is characterized by the asymptotics $\Phi \rightarrow \exp (i \vec{\alpha} \cdot \vec{\xi}), z \rightarrow$ $-\infty$, while $\Psi_{ \pm}(\vec{x}, z, \lambda \vec{\alpha})$ are analytic respectively in the upper and lower halves of the $\lambda$ plane, with asymptotics:

$$
\Psi_{ \pm}=e^{i \vec{\alpha} \cdot \vec{\xi}}\left(1+\frac{i \vec{\alpha} \cdot \vec{Q}_{ \pm}(\vec{x}, z)}{\lambda}+O\left(\lambda^{-2}\right)\right)
$$

From equations (57) and (33) it follows that

$$
\Psi_{ \pm}(\vec{x}, z, \lambda, \vec{\alpha})=\Phi(\vec{x}, z, \lambda, \vec{\alpha})+e^{i \vec{\alpha} \cdot \vec{\varphi}(\vec{x}, z, \lambda)}\left(e^{i \vec{\alpha} \cdot \vec{\chi}_{ \pm}(\vec{\varphi}(\vec{x}, z, \lambda), \lambda)}-1\right) .
$$

The last term of this equality is clearly equal to its anti-Fourier transform:

$$
e^{i \vec{\alpha} \cdot \vec{\varphi}}\left(e^{i \vec{\alpha} \cdot \vec{\chi} \pm(\vec{\varphi}, \lambda)}-1\right)=\int_{\mathbb{R}^{2}} d \vec{\beta} K_{ \pm}(\vec{\alpha}, \vec{\beta}, \lambda) e^{i \vec{\beta} \cdot \vec{\varphi}}, \quad \vec{\xi} \in \mathbb{R}^{2},
$$

where

$$
K_{ \pm}(\vec{\alpha}, \vec{\beta}, \lambda) \equiv \int_{\mathbb{R}^{2}} \frac{d \vec{\xi}}{(2 \pi)^{2}} e^{i(\vec{\alpha}-\vec{\beta}) \cdot \vec{\xi}}\left[e^{i \vec{\alpha} \cdot \vec{\chi} \pm(\vec{\xi}, \lambda)}-1\right] .
$$

Then, using (57), the last term in the RHS of (59) becomes a linear expression in terms of $\Phi$, and equations (59) give the linear representations the analytic eigenfunctions $\Psi_{ \pm}$in terms of the Jost eigenfunction $\Phi$ :

$$
\Psi_{ \pm}(\vec{x}, z, \lambda, \vec{\alpha})=\Phi(\vec{x}, z, \lambda, \vec{\alpha})+\int_{\mathbb{R}^{2}} d \vec{\beta} K_{ \pm}(\vec{\alpha}, \vec{\beta}, \lambda) \Phi(\vec{x}, z, \lambda, \vec{\beta}) .
$$

Multiplying the equations (62) + and (62) - by $\exp (-i \vec{\alpha} \cdot \vec{\xi})$, subtracting 1 , applying respectively $\hat{P}_{-}$and $\hat{P}_{+}$, and adding the resulting equations, one obtains the following linear integral equation for $\Phi$ :

$$
\begin{aligned}
& \Phi(\lambda, \vec{\alpha})+\frac{1}{2 \pi i} \int_{\mathbb{R}} \frac{d \lambda^{\prime}}{\lambda^{\prime}-(\lambda+i \epsilon)} \int_{\mathbb{R}^{2}} d \vec{\beta} K_{-}\left(\vec{\alpha}, \vec{\beta}, \lambda^{\prime}\right) \Phi\left(\lambda^{\prime}, \vec{\beta}\right) e^{i \alpha_{1}\left(\lambda^{\prime}-\lambda\right) z}- \\
& -\frac{1}{2 \pi i} \int_{\mathbb{R}} \frac{d \lambda^{\prime}}{\lambda^{\prime}-(\lambda-i \epsilon)} \int_{\mathbb{R}^{2}} d \vec{\beta} K_{+}\left(\vec{\alpha}, \vec{\beta}, \lambda^{\prime}\right) \Phi\left(\lambda^{\prime}, \vec{\beta}\right) e^{i \alpha_{1}\left(\lambda^{\prime}-\lambda\right) z}=e^{i \vec{\alpha} \cdot \vec{\xi}},
\end{aligned}
$$

in which we have omitted, for simplicity, the parametric dependence on $(\vec{x}, z)$ of $\Phi$. Once $\Phi$ is reconstructed from (63) and, via (62), $\Psi_{ \pm}$are also known, 
the potentials are reconstructed in the usual way from the asymptotics (58) of $\Psi_{ \pm}$.

We finally observe that the reality constraints for the eigenfunctions $\Phi, \Psi_{ \pm}$and for the data $K_{ \pm}$read, for $\lambda \in \mathbb{R}$,

$$
\begin{aligned}
& \overline{\Phi(\vec{x}, z, \lambda, \vec{\alpha})}=\Phi(\vec{x}, z, \lambda,-\vec{\alpha}), \quad \overline{\Psi_{+}(\vec{x}, z, \lambda, \vec{\alpha})}=\Psi_{-}(\vec{x}, z, \lambda,-\vec{\alpha}), \\
& \overrightarrow{K_{+}(\vec{\alpha}, \vec{\beta}, \lambda)}=K_{-}(-\vec{\alpha},-\vec{\beta}, \lambda) \text {, }
\end{aligned}
$$

while the $t$-evolution of $K_{ \pm}$is given by:

$$
K_{ \pm}(\vec{\alpha}, \vec{\beta}, \lambda, t)=K_{ \pm}(\vec{\alpha}, \vec{\beta}, \lambda, 0) e^{i \lambda\left(\alpha_{2}-\beta_{2}\right) t}
$$

\section{Dressing}

Dressing schemes corresponding to the three inverse problems presented in this paper can always be formulated (see, f.i., [16] for a general treatment). Here we restrict our attention to that corresponding to the $\mathrm{RH}$ inverse problem of Section 5.1, but for the eigenfunctions $\vec{\pi}_{ \pm}$defined in (43). The proof, quite standard in the dressing philosophy, is left to the reader.

Consider the following nonlinear $R H$ problem on the real $\lambda$-axis:

$$
\vec{\pi}_{+}=\vec{\pi}_{-}+\overrightarrow{\breve{R}}\left(\vec{\pi}_{-}, \lambda\right) \equiv \overrightarrow{\mathcal{R}}\left(\vec{\pi}_{-}, \lambda\right), \quad \lambda \in \mathbb{R}
$$

for the functions $\vec{\pi}_{+}(\vec{x}, z, t, \lambda)$ and $\vec{\pi}_{-}(\vec{x}, z, t, \lambda)$, analytic respectively in the upper and lower halves of the complex plane $\lambda$, with asymptotics:

$$
\vec{\pi}_{ \pm}(\vec{x}, z, \lambda)=\left(\begin{array}{c}
x-\lambda z \\
y-\lambda t
\end{array}\right)+\frac{\vec{Q}_{ \pm}(\vec{x}, z, t)}{\lambda}+O\left(\lambda^{-2}\right) . \quad|\lambda|>>1,
$$

where the vector $\overrightarrow{\mathscr{R}}\left(\vec{\pi}_{-}, \lambda\right)$ depends on $(\vec{x}, z, t)$ only through $\vec{\pi}_{-}$. Assume also the unique solvability of the RH problem (66) and of its linearized version

$$
\begin{aligned}
& \vec{\nu}_{+}=\vec{\nu}_{-}+\rho\left(\vec{\pi}_{-}, \lambda\right) \vec{\nu}_{-}, \quad \lambda \in \mathbb{R}, \\
& (\rho)_{i j}(\vec{\zeta}, \lambda) \equiv \frac{\partial \check{R}_{i}(\vec{\zeta}, \lambda)}{\partial \zeta_{j}} .
\end{aligned}
$$

Then $\vec{\pi}_{ \pm}$are solutions of $\hat{L}_{1} \vec{\pi}_{ \pm}=\hat{L}_{2} \vec{\pi}_{ \pm}=\overrightarrow{0}$, where $\hat{L}_{1}, \hat{L}_{2}$ are defined in (14) with:

$$
\vec{u}_{1}(\vec{x}, z, t)=-\vec{Q}_{ \pm x}, \quad \vec{u}_{2}(\vec{x}, z, t)=-\vec{Q}_{ \pm y} .
$$


It follows that the potentials $\vec{u}_{1,2}$ are solutions of the nonlinear system of PDEs (13). If, in addition, the spectral data satisfy the Hamiltonian constraint

$$
\check{R}_{1 \zeta_{1}}+\check{R}_{2 \zeta_{2}}+\left\{\check{R}_{1}, \check{R}_{2}\right\}_{\vec{\zeta}}=0,
$$

then the potentials $\vec{u}_{1,2}$ satisfy the heavenly equation (11). At last, the reality constraints

$$
\overrightarrow{\overrightarrow{\mathcal{R}}} \overline{(\overrightarrow{\mathcal{R}}(\overline{\vec{\zeta}}, \lambda)}, \lambda)=\vec{\zeta}, \quad \forall \vec{\zeta}, \quad \lambda \in \mathbb{R}
$$

imply that $\overline{\vec{\pi}_{+}}=\vec{\pi}_{-}$, and the reality of the potentials: $\vec{u}_{1}, \vec{u}_{2} \in \mathbb{R}^{2}$.

Acknowledgements. The visit of SVM to Rome was supported by the INFN grant 2005 and by the RFBR grant 04-01-00508.

\section{References}

[1] J. F. Plebanski, "Some solutions of complex Einstein equations", J. Math. Phys. 16, 2395-2402 (1975).

[2] C. N. Yang and R. L. Mills, "Conservation of isotopic spin and isotopic gauge invariance", Phys. Rev 96, 191-195 (1954).

[3] V. E. Zakharov and A. B. Shabat, Functional Anal. Appl. 13, 166-174 (1979).

[4] V. E. Zakharov, S. V. Manakov, S. P. Novikov and L. P. Pitaevsky, Theory of solitons, Plenum Press, New York, 1984.

[5] M. J. Ablowitz and P. A. Clarkson, Solitons, nonlinear evolution equations and Inverse Scattering, London Math. Society Lecture Note Series, vol. 194, Cambridge University Press, Cambridge (1991).

[6] S. Chakravarty, L. Mason and E. T. Newman, "Canonical structures on anti-selfdual four manifolds and the diffeomorphism group", J. Math. Phys. 32, 1458-1464, (1991).

[7] M. Dunajski and L. J. Mason, "Hyper-Kähler hierachies and their twistor theory", Comm. Math. Phys. 213, 641-672 (2000). "Twistor theory of hyper-Kähler metrics with hidden symmetries", J. Math. Phys., 44, 34303454 (2003). 
[8] M. Dunajski, L. J. Mason and P. Todd, "Einstein-Weyl geometry, the dKP equation and twistor theory", J. Geom. Phys. 37 63-93 (2001).

[9] F. Neyzi, Y. Nutku and M. B. Sheftel, "A multi-hamiltonian structure of the Plebanski's second heavenly equation"; J. Phys. A: Math. Gen. 38 8473- (2005).

[10] M. Manas and L. Martinez Alonso, "A hodograph transformation which applies to the heavenly equation"; arXiv:nlin.SI/0209050 v1.

[11] L. V. Bogdanov and B. G. Konopelchenko, "On the $\bar{\partial}$-dressing method applicable to heavenly equation"; Phys. Lett. A 345 (2005) 137-143; "On the heavenly equation hierarchy and its reductions", arXiv:nlin.SI/0512074.

[12] S. V. Manakov and P. M. Santini: "Inverse scattering problem for vector fields and the heavenly equation"; http://arXiv:nlin.SI/0512043.

[13] S. V. Manakov and V. E. Zakharov, "Three-dimensional model of relativistic-invariant field theory, integrable by the inverse scattering transform"; Letters in Mathematical Physics 5, 247-253 (1981).

[14] S. V. Manakov, "The inverse scattering transform for the time - dependent Schrödinger operator and Kadomtsev-Petviashvili equation", Physica 3D, 420-427 (1981).

[15] R. Courant and D. Hilbert, Methods of Mathematical Physics; Vol. II: Partial Differential Equations, by R. Courant, Interscience Publishers, J. Wiley and sons, New York, 1962.

[16] L.V.Bogdanov and S.V.Manakov, J.Phys.A:Math.Gen. 21, L537 (1988) 